From No. 15 we get the theorem:-If from any point $M$ on a conic, a tangent be drawn meeting a confocal in $\mathrm{N}$, the product of the perpendicular from the centre on the tangent at $\mathbf{N}$ by the intercept on the normal at $\mathbf{N}$ between the tangents at $\mathbf{M}$ and $\mathbf{N}$ is constant.

Mr J. S. MackaY gave a synopsis of Frans Schooten's "Geometry of the Rule," as it is contained in the second book of the Exercitationes Mathematicae, Leyden, 1657.

Mr P. Alexander contributed a note on the two definite integrals

$$
\int_{0}^{\infty} \sin n x d x \text { and } \int_{0}^{\infty} \cos n x d x
$$

Sixth Meeting, April 10th, 1885.

A. J. G. Barclay, Esq., M.A., President, in the Chair.

Note on the evaluation of functions of the Form $0^{\circ}$.

By T. B. Sprague, M.A., F.R.S.E.

Let $f(t), \phi(t)$, be two functions of $t$, such that they both vanish with $t$, that is, $f(0)=0, \phi(0)=0$; and put

$$
z=\{f(t)\} \Phi t \text {. }
$$

Then, in order to find the limiting value of $z$ when $t=0$, we proceed as follows:-

$$
\log z=\phi(t) \cdot \log f(t)=\frac{\log f(t)}{\frac{1}{\phi(t)}}
$$

This fraction takes the form $-\frac{\infty}{\infty}$ when $t=0$, and we therefore have 


$$
\begin{aligned}
\operatorname{Ltlog} z & =\operatorname{Lt} \frac{\log [f(t)]}{\frac{1}{\phi(t)}}=\operatorname{Lt} \frac{\frac{f^{\prime}(t)}{f(t)}}{-\frac{\phi^{\prime}(t)}{\{\phi(t)\}^{2}}} \\
& =-\operatorname{Lt} \frac{f^{\prime}(t)}{\phi^{\prime}(t)} \cdot \begin{array}{c}
{[\phi(t)]^{2}} \\
f(t)
\end{array} .
\end{aligned}
$$

In the limit, $\frac{\phi(t)}{f(t)}$ takes the form $\frac{0}{0}$; and the value of it is the same as that of $\frac{\phi^{\prime}(t)}{f^{\prime}(t)}$. If this value is finite, say $=a$, then

$$
\mathrm{Lt} \frac{f^{\prime}(t)}{\phi^{\prime}(t)} \cdot \frac{\phi(t)}{f(t)} \cdot \phi(t)=\mathbf{L t} \frac{a}{a} \cdot \phi(t)=0 .
$$

Hence, in the limit, $\log z=0$, and $z=1$.

If, however, $\frac{\phi(0)}{f(0)}$ is either intinitely small or infinitely large, the above expression takes the forms $\frac{0}{0}$ and $\frac{\infty}{\infty} \times 0$, respectively; and we are unable without further investigation to draw any conclusion as to the limiting value of $z$.

As a particular case, suppose that $\phi(t)=t^{2}, f(t)=t$, so that $\frac{\phi(t)}{f(t)}=t$, and $\frac{\phi(0)}{f(0)}=0$; then

$$
\mathrm{Lt} \frac{f^{\prime}(t)}{\phi^{\prime}(t)} \cdot \frac{[\phi(t)]^{9}}{f(t)}=\mathrm{Lt} \frac{1}{2 t} \cdot \frac{t^{4}}{t}=\mathrm{Lt} \frac{t^{2}}{2}=0 ;
$$

so that in this case also, $\mathrm{Lt} z=1$.

$$
\text { Next suppose } \phi(t)=t, f(t)=t^{2} \text {; so that } \frac{\phi(t)}{f(t)}=\frac{1}{t} \text {, and } \frac{\phi(0)}{f(0)}=\infty \text {; }
$$
then $\mathrm{Lt} \log z=\mathrm{Lt} \frac{2 t}{1} \cdot \frac{t^{2}}{t^{2}}=\mathrm{Lt} 2 t=0$; and again $\mathrm{Lt} z=1$.

In every case we have as yet considered, the limit of $z$ is 1 ; and we can prove that it has the same value whenever $f(t)$ and $\phi(t)$ are ordinary algebraic functions; that is to say, when neither of them is a transcendental function. In this case, since $f(0)=0$ and $\phi(0)=0$, $f(t)$ and $\phi(t)$ must both be capable of expansion in series containing only positive powers of $t$, so that

$$
\begin{aligned}
& f(t)=a t^{m}+b t^{n}+\ldots \ldots \\
& \phi(t)=a t^{\mu}+\beta t^{\nu}+\ldots \ldots
\end{aligned}
$$

where the indices are all positive, and $t^{m}, t \mu$, are the lowest powers of $t$ in the two series respectively, then 


$$
\begin{aligned}
f^{\prime}(t) & =m a t^{m-1}+n b t^{n-1}+\ldots \ldots \\
\phi^{\prime}(t) & =\mu a t^{\mu}-1+v \beta t^{\mu-1}+\ldots \ldots
\end{aligned}
$$

and $\quad \operatorname{Lt} \frac{f^{\prime}(t)}{\phi^{\prime}(t)} \cdot \frac{[\phi(t)]^{2}}{f(t)}=\operatorname{Lt} \frac{t f^{\prime}(t)}{f(t)} \cdot \frac{\phi(t)}{t \phi^{\prime}(t)} \cdot \phi(t)=\frac{m}{\mu} \times 0=0$;

so that, as before, $\operatorname{Lt} z=1$.

We can furthermore prove that, whenever $\frac{\phi(0)}{f(0)}$ is zero, the limit of $z$ is 1 . For

$$
\log x=\phi t \log (f t)=\frac{\phi t}{f t} \times f t \log (f t),
$$

$$
\text { and Lt } \log z=\mathrm{Lt} \frac{\phi t}{\overrightarrow{f t}} \times \mathrm{Lt} f t \log (f t) \text {. }
$$

But Lt $f t \log (f t)=\mathrm{Lt} \frac{\log (f t)}{\frac{1}{f t}}=\mathrm{Lt} \frac{\frac{f^{\prime} t}{f t}}{-\frac{f^{\prime \prime} t}{(f t)^{2}}}=-\mathrm{Lt} f t$

$$
=0 \text {. }
$$

Hence Lt $\log z=0 \times 0=0$, and Lt $z=1$. The same proof niay be employed in the case when $\frac{\phi(0)}{f(0)}$ is finite.

That we must not from these results conclude that the limit of $z$ is always 1 , at once appears from the fact that, by giving a suitable form to $\phi(t)$, we may make the limit have any finite positive value, say c. In fact, suppose that $f(t), \phi(t)$, are such functions that for all values of $t$ $\{f(t)\} \phi(t)$ is constant and $=c$.

Then $\phi(t)=\frac{\log c}{\log f(t)} ;$ and, if $f(0)=0, \phi(0)=\frac{\log c}{-\infty}=0$;

or $f(t), \phi(t)$, both vanish with $t$. In this case we have for all values of $t, z=(f t) \phi t=c$; and the same is still true in the limit when $t=0$.

The value of $\frac{\phi(0)}{f(0)}$ is now infinite; for

$$
\begin{aligned}
\text { Ltt } \frac{\phi(t)}{f(t)} & =\operatorname{Lt} \frac{\log c}{f(t) \log f(t)}=\log c \operatorname{Lt} \frac{\frac{1}{f(t)}}{\log f(t)} \\
& =\log c \operatorname{Lt} \frac{-\frac{f^{\prime}(t)}{\left.f^{\prime}(t)\right]^{2}}}{f(t)}=-\log c \operatorname{Lt} \frac{1}{f(t)} \\
& = \pm \infty ;
\end{aligned}
$$


the upper or lower sign being used according as $\log c$ is negative or positive.

It is easy to assign other forms to $\phi(t)$ that will make the limit of $a$ different from 1. Taking the expression

$$
\phi(t)=\frac{\log c}{\log f(t)},
$$

if we add to both numerator and denominator quantities which ultimately vanish in comparison with them, the limit of $z$ will still be $c$. Thus, let us put ( $n$ being positive and not $<1$ ),

$$
\phi(t)=\frac{\log c}{t^{n}+\log f(t)} ;
$$

$$
\begin{array}{cc}
\text { then } & \frac{1}{\phi(t)}=\left\{t^{n}+\log f(t)\right\} \div \log c ; \\
\therefore & -\frac{\phi^{\prime}(t)}{[\phi(t)]^{2}}=\left\{n t^{n-1}+\frac{f^{\prime}(t)}{f(t)}\right\} \div \log c ; \\
\text { and } & -\frac{f^{\prime}(t)}{f(t)} \cdot \frac{[\phi(t)]^{2}}{\phi^{\prime}(t)}=\frac{f^{\prime}(t)}{f(t)} \cdot \frac{\log c}{n t^{n-1}+\frac{f^{\prime}(t)}{f(t)}}=\frac{\log c}{n t^{n-1} \frac{f(t)}{f^{\prime}(t)}+1} .
\end{array}
$$

Now, if $f(0)=0$, then $\frac{f(0)}{f^{\prime}(0)}=0$; for,tbeing the abscissa ON,(Fig. 16,17) and $f(t)$ the ordinate $\mathrm{PN}$, of a curve which passes through the origin, $\frac{f^{\prime}(t)}{f^{\prime}(t)}$ is the subtangent $\mathrm{TN}$, which evidently vanishes when $\mathrm{P}$ moves up to the origin, that is, when $t=0$.

Hence $-\mathrm{Lt} \frac{f^{\prime}(t)}{f(t)} \cdot \frac{[\phi(t)]^{2}}{\phi^{\prime}(t)}=\log c$; or, in the limit, $\log z=\log c$, and $z=c$.

It will be found that, as in the last case, $\frac{\phi(0)}{f(0)}=-\infty$, if $c>1$; and $=+\infty$, if $c<1$.

If we next desire to give $\phi(t)$ such a form that the limit of $z$ will be zero, we must examine what takes place when we suppose $c$ to become indefinitely small. In this case loge becomes indefinitely large, and negative; but since $\phi(t)$, which $=\frac{\log c}{\log f(t)}$, is to vanish with $t$, $\log c$ must vanish in comparison with $\log f(t)$. We must therefore substitute for $\log c$ a function which, when $t=0$, vecomes $-\infty$, but vanishes in comparison with $\log f(0)$. Such a function is $-\log \{-\log f(t)\}$. We will put, then, $n$ being positive,

$$
\phi(t)=\frac{-\log \{-\log f(t)\}}{m t^{n}+\log f(t)} .
$$


Hence $\quad \log z=\phi(t) \log f(t)=\frac{-\log \{-\log f(t)\}}{m t^{n}+\log f(t)} \cdot \log f(t)$

$$
=\frac{-\log \{-\log f(t)\}}{\frac{m t^{n}}{\log f(t)}+1}
$$

whence Lt $\log z=-\infty$, and It $z=0$. In this case

$$
\mathrm{L} t \frac{\phi(t)}{f(t)}=-\mathrm{Lt} \frac{\log \{-\log f(t)\}}{f(t)\left[m t^{n}+\log f(t)\right]}=\frac{-\infty}{-0}=+\infty ;
$$

since $m t^{n}$ vanishes in comparison with $\log f(t)$, and $\operatorname{Lt} f(t) \cdot \log f(t)=-0$.

Similarly, if we put

$$
\phi(t)=\frac{+\log \{-\log f(t)\}}{m t^{n}+\log f(t)},
$$

we shall get $\mathrm{Lt} z=\infty$, and $\mathbf{L t} \frac{\phi(t)}{f(i)}=-\infty$.

We have thus proved that when $\frac{\phi(0)}{f(0)}$ is either zero or finite, whether positive or negative, the limit of $z$ (or $\left.(f 0) \phi_{0}\right)$ is $=1$; and when $\frac{\phi(0)}{f(0)}=+\infty$, the limit may have any value from 0 to 1 ; and when $\begin{aligned} & \phi(0) \\ & f(0)\end{aligned}=-\infty$, any value from 1 to $\infty$.

It will help us to understand these results better if we study the form of the surface, $z=x^{y}$. Giving to $x$ any finite positive value, $+a$, and to $y$ any finite value, $b$, which may be either positive or negative, we get-one real and positive value of $z$, namely $a^{b}$; and the question we have been considering is the determination of the value of $z$ when both $a$ and $b$ simultaneously diminish according to a given law, and ultimately vanish together; or in geometrical language, the determination of the point or points, where the z-axis cuts the surface. We consider only positive values of $x$, because functions of the form $(-a)^{b}$ will not give us a continuous series of real values as $b$ varies continuously; and we neglect all the negative values of $z$, that arise from giving to $b$ such values as $\frac{1}{2}, \frac{3}{3}$, dc. ; or, in general, $\frac{2 m+1}{2 n}$; for these values also do not form a continuous series. Reverting to our old supposition that $x=f(t), y=\phi(t), f(0)=0$, and $\phi(0)=0$, then any value of $t$ determines a point in the $x y$ plane; and the two equations together determine a curve in that plane passing through the origin. This curve may be considered as the projection 
of a curve on the surface; and if we suppose $t$ to be diminished until it vanishes, and then determine the limiting value of $z$, this is equivalent to determining what point on the $x$-axis we shall arrive at, if we travel to it along an assigned line on the surface. When we proved above that, if $\frac{\phi(0)}{f(0)}$ is finite or zero, then $z_{0}=1$, we virtually prove that, if we travel along any line on the surface, the projection of which on the $x y$ plane passes through the origin and does not touch the $y$-axis, we shall always arrive at the point on the $z$-axis at a distance $=1$ from the origin. When we next proved that, if $f(t), \phi(t)$, are algebraic functions, the value of $z_{0}$ is still $=1$, although $\frac{\phi(0)}{f(\overline{0})}$ is infinite, we virtually proved that, if we travel along any curve on the surface, the projection of which is any algebraic curve passing through the origin and touching the $y$-axis, we still arrive at the same point. We have also proved that, by proceeding along a suitable curve on the surface, we may arrive at any point we please on the $z$-axis ; but in this case, the projection of the curve on the $x y$ plane, must have contact of an infinitely high order with the $y$-axis.

In order further to elucidate the subject, I will now consider the nature of the sections of the surface made by the plane, $z=c$, for different positive values of $c$. The equation to the projection of this section on the $x y$ plane, is $x y=c$; whence $y=\frac{\log c}{\log x}$. When $c<1$, the curve represented by this equation is of the form shown in Fig. 18, where the positive part of the $y$-axis is drawn downwards because this is more convenient than the usual position, when we desire to picture to ourselves the various sections as arranged on the surface, and thus obtain a conception of the configuration of the latter. The curve passes through the origin and touches the positive part of the $y$-axis; and the line $x=1$ is an asymptote. If we put $y=1$, we get $x=c$; and if $y=-1$, we get $x=\frac{1}{c}$. Hence, if we make in the figure, $\mathrm{OB}=\mathrm{O} b=\mathrm{OA}=1$, and draw $\mathrm{BP}, b p$, parallel to the $x$-axis and meeting the curve in $\mathrm{P}, p$, and then draw the perpendiculars $\mathrm{PD}, p d$; we have $\mathrm{OD}=c$, and $\mathrm{O} d=\frac{1}{c}$.

When $c>1$, the curve has the same form but it lies on the other side of the $x$-axis; and if the new value, of $c$ (say $c_{1}$ ) is the reciprocal 
of the old one, the new curve will be the reflection of the old one on the $x$-axis.

We have seen that, whatever positive value we give to $c$, other than unity, the curve in Fig. 18 passes through the origin; and we conclude that every point on the positive part of the z-axis, other than the point for which $z=1$, lies on the surface. The excepted point for which $z=1$ requires special consideration; in this case, we have $x^{y}=1$, and we see that when $x=1$ this is satisfied by any value of $y$; hence every point on the line determined by $x=1, z=1$ lies on the surface. The equation is also satisfied by putting for $x$ any finite positive quintity and making $y=0$; in other words, every point on the positive part of the line, $y=0, z=1$, lies on the surface; but we have still to consider the point $(001)$.

The best way of examining the form of the surface in the neighbourhood of this point, is to study the changes in the section of the surface by the plane $z=c$, as $c$ gradually approaches unity. For this purpose, we may neglect the part of the curve to the right of the asymptote. It is easily found that the coordinates of the point of inflection are $x=\epsilon^{-2}=\cdot 1353$, and $y=-\frac{1}{2} \log c$; and that the equation to the tangent at that point is

$$
\frac{x}{e^{-2}}+\frac{4 y}{\log c}+1=0 .
$$

Hence, if we take $\mathrm{OE}=\mathrm{OF}=\cdot 1353$ (Fig. 19), OA as before being unity, and draw $\mathrm{FH}$ parallel to $\mathrm{O} y$, the points of inflection of the various sections will all lie in $\mathrm{EH}$, and the tangents at them will all pass through the point $F$. We are now able to see that, as $c$ increases up to 1 , the curve of section gradually approximates to the straight lines $\mathrm{OA}$ and $\mathrm{AG}$; but, however close the curve is to $\mathrm{OA}$, so long as it does not actually coincide with $O A$, it touches $O y$. It is also easy to see that, when $c>1$, the curves lie on the negative side of the $x$-axis, and all touch the negative part of the $y$-axis.

These gometrical results show that when $x$ and $y$ both vanish, if the ultimate value of the ratio $y: x$ is either zero or finite, whether positive or negative, then the ultimate value of $x^{y}$ is unity. Let $\mathrm{PO}$ be the projection on the $x y$ plane, of the path of the moving point on the surfice, and suppose that the surface is cut by an indefinitely large number of planes parallel to the $x y$ plane, then, as $t$ recrives different vaiues, the path of the moving point on the surface will generally cut these curves of section; and the same will be true of 
the projections of the path and of the sections on the $x y$ plane; and the last of these curves which the path meets, will determine the ultimate value of $z$. Now, since all the projections of the sections touch the $y$-axis at the origin, if OP, the projection of the path of the moving particle, does not touch the $y$-axis at the origin, it must, as it approaches the origin, cut the projections of all the sections in the neighbourhood of the origin, until it meets at the origin, the line OA, which is the projection of the section for which $z=1$. Hence, if OP does not touch the $y$-axis at the origin, or if the ultimate value of $y: x$ for of $\phi(t): f(t)\}$ is not $\pm \infty$, then the ultimate value of $z$ is unity. This quite agrees with what was proved in the earlier part of this paper.

Dr Thomas Muir has furnisht me with the following references to papers which $I$ have not myself seen :-

Johnson, W. W. On the expression $0^{\circ}$. Analyst III, pp. 118-121 (1876).

Franklin, F. Note on indeterminate exponential forms. American Journal of Mathematics I, pp. 368-9 (1878).

\section{The so-called Simson line.}

\section{By John Alison, M.A.}

This paper is an attempt to collect and arrange some of the propositions regarding the so-called Simson line, contained in various Mathematical Treatises and Journals. Proofs have been altered, or new ones substituted to suit the arrangement.

$\S 1$. Figure 20. If from any point on the circumference of a circle perpendiculars be drawn to the sides of an inscribed triangle, the feet of these perpendiculars are collinear. [The lixe on which they lie is known as the Simson line; but it is doubtful if Simson knew it. See Nature, Vol. XXX. p. 635.]

$\S 2$. There are two theorems of which the preceding may be regarded as a particular case.

(a). If lines be drawn from any point on the circumfirence of a circle, making equal angles in the same direction with the three sides of an inscribed triangle, the points of intersection of these lines with 